\title{
Dual-layer collimator for improved spatial resolution in SPECT with CZT cameras: An analytical and Monte Carlo study
}

This paper was downloaded from TechRxiv (https://www.techrxiv.org).

\section{LICENSE}

CC BY 4.0

SUBMISSION DATE / POSTED DATE

$15-10-2021 / 18-10-2021$

\section{CITATION}

walrand, stephan; Boutaghane, Nasreddine; Hesse, Michel; Bouzid, Boualem; Hospital, Habib Zaidi - Geneva University; Jamar, François (2021): Dual-layer collimator for improved spatial resolution in SPECT with CZT cameras: An analytical and Monte Carlo study. TechRxiv. Preprint.

https://doi.org/10.36227/techrxiv.16817368.v1

$\mathrm{DOI}$

10.36227/techrxiv.16817368.v1 
Detailled table 1:

\begin{tabular}{|l|l|l|l|l|l|l|l|l|l|l|}
\hline $\mathrm{I}_{1}-\mathrm{I}_{2}[\mathrm{~mm}](\mathrm{MC})$ & $45-0.00$ & $40-0.90$ & $35-4.57$ & $30-7.75$ & $25-10.75$ & $20-12.95$ & $15-14.20$ & $10-15.30$ & $5-17.00$ & $0-19.20$ \\
\hline $\mathrm{g} \times 10^{4}$ (eq. 10 or 18) & 1.75 & 1.76 & 1.76 & 1.78 & 1.78 & 1.77 & 1.77 & 1.77 & 1.74 & 1.74 \\
\hline FWHM (MC) [mm] & 7.34 & 7.26 & 6.93 & 6.75 & 6.59 & 6.52 & 6.66 & 6.84 & 6.84 & 6.67 \\
\hline FWHM (eq. 23) [mm] & 7.37 & 7.32 & 7.00 & 6.80 & 6.63 & 6.55 & 6.69 & 6.87 & 6.85 & 6.67 \\
\hline
\end{tabular}

